\title{
Influences observed on incidence and reporting of medication errors in anesthesia
}

\section{Influences observées sur l'incidence et le signalement des erreurs médicamenteuses en anesthésie}

\author{
Lebron Cooper, MD • Neil DiGiovanni, MD • \\ Lucy Schultz, RN • Ashley M. Taylor, PharmD • \\ Bobby Nossaman, MD
}

Received: 16 December 2011/ Accepted: 13 March 2012/Published online: 31 March 2012

(C) Canadian Anesthesiologists' Society 2012

\begin{abstract}
Background Medication errors are a common occurrence during the conduct of anesthesia (one in 113-450 patients). Several factors contribute to medication errors in anesthesia, including experience of the anesthesia provider, severity of comorbidities, and type of procedure. The inexperience of anesthesia providers-in-training also leads
\end{abstract}

\begin{abstract}
Author contributions Lebron Cooper, Neil DiGiovanni, and Bobby Nossaman participated in drafting the manuscript. Lebron Cooper and Bobby Nossaman have seen the original study data, reviewed the analysis of the data, and revised the final manuscript. Neil DiGiovanni contributed to the initial study design, including design of the reporting tool used in this study. He was responsible for entering and maintaining the study database and for statistical analysis of the data. Lucy Schultz and Ashley M. Taylor assisted with the collection and analysis of the data. Ashley M. Taylor was employed as a Clinical Pharmacy Specialist at Ochsner Health System in New Orleans, Louisiana when the data collection occurred, and data analysis occurred while she was employed as a Clinical Assistant Professor of Pharmacy at Xavier University of Louisiana College of Pharmacy. She was also involved during multiple steps of the research process and was a co-author for the attached project.
\end{abstract}

This report was previously presented, in part, at the International Anesthesia Research Society and the American Society of Anesthesiologists and was the subject of an article in Anesthesiology News 2009; 35(6).

Electronic supplementary material The online version of this article (doi:10.1007/s12630-012-9696-6) contains supplementary material, which is available to authorized users.

L. Cooper, MD · N. DiGiovanni, MD · L. Schultz, RN ·

B. Nossaman, MD

Department of Anesthesiology, Ochsner Clinic Foundation, Ochsner Health System, New Orleans, LA, USA

A. M. Taylor, PharmD

Department of Pharmacy, Ochsner Clinic Foundation, Ochsner

Health System, New Orleans, LA, USA to increased error rates. This prospective observational study repeats and extends previous work by Webster et al. and Llewellyn et al. examining the role of comorbidities, type of case, and level of provider experience on the incidence of medication errors.

Methods After Institutional Review Board review and exemption from informed consent, medication error reporting forms were attached to every anesthetic record during a six-month period. All providers were asked to return the forms for every anesthetic, on a strictly voluntary and anonymous basis, and to record the occurrence of medication errors. If providers indicated that a medication error had occurred, additional details about the event were obtained anonymously.

Results There were 8,777 (83\%) responses obtained in a review of 10,574 case forms. A medication error was reported in 35 forms, with an additional 17 forms indicating a medication pre-error or near miss, resulting in 52 (0.49\%) errors/pre-errors or a reported incidence of 1:203 anesthetics. Most case types were observed to have a statistically significant increase in reported medication errors. Reported errors by type of anesthesia provider were categorized into anesthesia provider-in-training group and the experienced provider group. The anesthesia providerin-training group reported a twofold increase in the rate of errors, with the most frequently reported errors being incorrect dose and substitution.

\footnotetext{
L. Cooper, MD ( $\square)$

Department of Anesthesiology, University of Miami Hospital, 1400 NW 12th Ave, Suite 3155, Miami, FL 33136, USA e-mail: 1cooper@med.miami.edu
} 
Conclusion This study suggests that case type, American Society of Anesthesiologists' classification, and level of provider experience play a role on the rate of medication errors. The results of this study are in agreement with previously reported error rates.

\section{Résumé}

Contexte Les erreurs médicamenteuses surviennent fréquemment au cours de la réalisation d'une anesthésie (1 erreur pour 113 à 450 patients). Plusieurs facteurs contribuent aux erreurs médicamenteuses en anesthésie, notamment l'expérience de la personne assurant l'anesthésie, la gravité des comorbidités et le type d'intervention. L'inexpérience d'anesthésiologistes en formation conduit également à des erreurs plus fréquentes. Cette étude observationnelle prospective reprend et prolonge les travaux de Webster et coll. et de Llewellyn et coll. en analysant le rôle des comorbidités, du type de cas et du niveau d'expérience du professionnel sur l'incidence des erreurs médicamenteuses.

Méthodes Après analyse, le comité d'éthique de la recherche a levé l'exigence de consentement éclairé. Des formulaires de signalement d'erreurs médicamenteuses ont été joints à chaque dossier d'anesthésie pendant une période de six mois. Il était demandé à tous les professionnels de soins de renvoyer le formulaire pour chaque anesthésie sur une base volontaire et anonyme, en y inscrivant la survenue d'erreurs médicamenteuses. Si les professionnels indiquaient qu'une erreur médicamenteuse était survenue, des détails complémentaires sur l'événement étaient collectés de façon anonyme.

Résultats Il y a eu 8777 réponses obtenues à partir de l'analyse de 10574 formulaires de cas (soit $83 \%$ ). Une erreur médicamenteuse a été signalée dans 35 formulaires, tandis que 17 autres formulaires indiquaient une " erreur préalable » ou une "erreur évitée de peu », aboutissant à un total de $52(0,49 \%)$ erreurs/erreurs préalables, soit une incidence signalée de 1/203 anesthésies. Dans la plupart des types de cas on a observé une augmentation statistiquement significative des erreurs médicamenteuses signalées. Les erreurs signalées ont été classées selon deux groupes de professionnels d'anesthésie: le groupe des anesthésiologistes en formation et le groupe des anesthésiologistes expérimentés. Le groupe des anesthésiologistes en formation a signalé deux fois plus d'erreurs: les erreurs signalées les plus fréquentes concernaient les doses et les substitutions incorrectes.

Conclusion Cette étude suggère que le type de cas, la classification de l'American Society of Anesthesiologists et le niveau d'expérience de l'anesthésiologiste jouent un rôle dans la fréquence des erreurs médicamenteuses. Les résultats de cette étude concordent avec les fréquences d'erreur publiées précédemment.

\section{Introduction}

Medication errors/pre-errors are a common occurrence during the conduct of anesthesia, with a reported frequency of one out of every 113-450 anesthetics administered. ${ }^{1-5}$ Complex surgical procedures and patients with significant comorbidities often require therapeutic interventions with infrequently used medications utilized under dynamic conditions, all contributing to higher medication error rates when compared with cases with lower surgical complexity. ${ }^{6,7}$ The inexperience of trainees in the specialty may also lead to a higher frequency of medication errors in teaching programs, yet there are few data to support this claim. $^{8}$ Two prospective studies ${ }^{1,9}$ and one retrospective study ${ }^{4}$ have reported human factors contributing to medication errors/pre-errors during the conduct of anesthesia. Although Fasting and Gisvold reported that anesthesiologists have been involved in at least one medication error/ pre-error, most medication errors were deemed inconsequential. ${ }^{5}$ Factors associated with medication errors/ pre-errors occurring within the operating room include personal distractions; production pressure; inadequate communication between medical caregivers; exposure to new, unexpected, or unfamiliar medication vials or labels; and misread/look-alike medication vials or ampules. ${ }^{1,3,4,10}$

The aim of this prospective unblinded observational study was to determine whether the type of surgical case, the American Society of Anesthesiologists' (ASA) physical status classification, the level of experience of the anesthesia provider, or other factors impact the frequency and reporting of medication errors/pre-errors in a tertiary care academic teaching hospital. This study extends previous work by Webster et al. ${ }^{1}$ from 2001 and Llewellyn et al. ${ }^{3}$ from 2009.

\section{Methods}

Following Institutional Review Board approval as a study exempt from informed consent, this prospective observational study was conducted at a major tertiary teaching hospital located in the southern United States. The composition of the anesthesia group consisted of 35 full-time attending anesthesiologists, 21 anesthesiology resident physicians from the study hospital residency program, 50 certified registered nurse anesthetists (CRNAs), and ten student nurse anesthetists (SRNAs). Trainees in anesthesia (providers-in-training) were defined as anesthesiology residents (PGY2-PGY4) and SRNAs. Experienced providers were defined as those who have completed training, which included attending anesthesiologists and certified registered nurse anesthetists (CRNAs). Physician and non-physician providers had the same prescribing and 
administration privileges, with all anesthetics under continuous medical direction of an attending anesthesiologist.

Surgical case type encompassed the full spectrum of surgical care, including cardiac and solid-organ transplantation. Surgical case type was defined by procedure and then grouped into surgical service lines for comparison. The service lines chosen were ophthalmology (OPHTH), neurosurgery (NEURO), off-site sedation (OFFSITE), obstetrics/gynecology (OB/GYN), general surgery (GEN), orthopedic (ORTHO), otolaryngology (ENT), urology (URO), cardiovascular/ thoracic surgery (CVT), colorectal (CR), organ transplant (TRANS), peripheral vascular surgery (VASC), and pediatric surgery (PEDS). If identical surgical procedures were performed by two different service lines, the case type was defined by the specialty of the surgeon performing the procedure. The attending anesthesiologist obtained the preoperative history of patients' comorbidities, assigned the ASA classification score, and designed the anesthesia plan of care.

Medication error reporting forms were designed to elicit voluntary and anonymous responses concerning the occurrence, type, patient demographics, interventions, consequences, and perceived environmental factors that contributed to the error (Appendix, available as Electronic Supplemental Material). The reporting form used in this study is similar to the data collection tool utilized by Webster et al., ${ }^{1}$ but it was further modified to examine the role of surgical case type, ASA classification, and level of clinical experience on the incidence of medication errors/ pre-errors. All medication errors/pre-errors were classified as previously reported by Webster et al. ${ }^{1}$ and Bowdle ${ }^{2}$ (Table 1).

All anesthesia providers received multiple classroom education sessions about medication error/pre-error

Table 1 Error classification definitions (adapted from Webster et al. ${ }^{1}$ and Bowdle ${ }^{2}$

\begin{tabular}{ll}
\hline Error Classification Definitions \\
\hline Pre-error/near-miss & $\begin{array}{l}\text { Any incident in which there was a } \\
\text { potential to become an error. }\end{array}$ \\
\hline Omission & $\begin{array}{c}\text { Drug not administered or } \\
\text { administered late. } \\
\text { Extra dose of intended drug given. } \\
\text { Repetition } \\
\text { Substitution } \\
\text { Incorrect drug administered instead of } \\
\text { intended drug. } \\
\text { Insertion } \\
\text { Drug administered which was not } \\
\text { intended at that time or at any stage. } \\
\text { Incorrect Dose } \\
\text { rate of infusion of the desired drug } \\
\text { administered. }\end{array}$ \\
$\begin{array}{l}\text { Incorrect Route/ } \\
\text { Inappropriate Drug } \\
\text { Administration }\end{array}$ & $\begin{array}{c}\text { Administered intravenous instead of } \\
\text { intramuscular; use of a multi-dose } \\
\text { vial on more than one patient. }\end{array}$ \\
\hline
\end{tabular}

Table 2 Patient harm classification/consequences of error

Minor Morbidity with Reversible Inadvertent overdose reversed Harm - (Not requiring escalation prior to permanent harm of care)

\begin{tabular}{lc}
$\begin{array}{c}\text { Major Morbidity with Reversible } \\
\text { Harm) - (Requiring escalation } \\
\text { of care) }\end{array}$ & $\begin{array}{c}\text { Increased monitoring, insertion } \\
\text { of PA catheter, TEE, or } \\
\text { prolonged PACU monitoring }\end{array}$ \\
$\begin{array}{c}\text { Major Morbidity with Irreversible } \\
\text { Harm - (Requiring escalation of } \\
\text { care) }\end{array}$ & $\begin{array}{c}\text { SIA, prolonged neurologic } \\
\text { Mortality }\end{array}$ \\
No Harm & $\begin{array}{c}\text { Death directly or indirectly } \\
\text { related to the error }\end{array}$ \\
\hline
\end{tabular}

PA = pulmonary artery; TEE = transesophageal echocardiography; PACU = postanesthesia care unit; $\mathrm{MI}=$ myocardial infarction; $\mathrm{CVA}=$ cerebrovascular accident

recognition and classification prior to the start of this study and again at four-week intervals. A key component of these no-fault or "systems-based" sessions was the guaranteed indemnification by departmental administration for the voluntary and anonymous reports of medication errors/preerrors collected in this study. The survey instrument was attached to every anesthesia record during a six-month period from August 20, 2007 to February 20, 2008. Anesthesia providers were asked to complete the medication error/pre-error reporting form for every anesthetic performed, whether or not a medication error or pre-error had occurred. If a provider indicated that an error or preerror had occurred, further details were elicited and entered on the survey instrument, including the provider's level of training, patient ASA classification, type of surgical case, patient harm classification/consequences of error (Table 2), as well as the provider's perception of environmental factors that may have led to the error. For all forms, the type of anesthesia provider, patient ASA classification, and data regarding the type of surgical case were obtained from the surgical informational system, if that information was not recorded by the anesthesia provider. The forms were collected daily and data were entered into an electronic database for statistical analysis.

Statistical analysis was performed using JMP ${ }^{\circledR}$ 6.0.0 software (SAS ${ }^{\circledR}$ Institute Inc., Cary, NC, USA). Categorical variables were presented as percentages, and differences between the groups were assessed using Chi square or Fisher's exact tests. Statistical significance was determined to be $P<0.05$.

\section{Results}

There were $8,777(83.0 \%)$ of a possible 10,574 anesthesia case forms returned. Of the 8,777 forms returned, 8,725 
indicated that no medication error/pre-error was recognized as having occurred. By eliciting negative responses, the response rate was monitored over time. There were 52 errors/pre-errors reported in 52 anesthetic cases; 35 of these were reported as errors and 17 were reported as preerrors or near misses. Two of the 52 errors/pre-errors occurred in the immediate postanesthesia period, while all others occurred during the pre-induction, induction, maintenance, or emergence phase of anesthesia. All surgical cases were included, whether elective or urgent/emergent. Only one error was reported in an emergency case, which occurred between 11:00 am and 3:00 pm. No errors were reported after 11:00 pm. The remainder of the errors/preerrors were reported to have occurred between 7:00 am and 11:00 pm. The response rate corresponds with a voluntarily reported medication error/pre-error rate of $0.49 \%(95 \%$ confidence interval, 0.37 to 0.64 ) or a reported occurrence rate of one out of every 203 anesthetics, based on the total number of anesthetics administered during the study period.

The voluntarily reported medication error/pre-error rate for each surgical case type was also determined (Table 3). There were no reports of medication errors/pre-errors in OPHTH or NEURO cases. All other surgical case types, including OFFSITE $(n=2)$, OB/GYN $(n=5)$, ENT $(n=4)$, GEN $(n=8)$, ORTHO $(n=9)$, URO $(n=5)$, CVT $(n=3)$, CR $(n=4)$, TRANS $(n=2)$, VASC $(n=5)$, and PEDS $(n=5)$, reported medication error/preerror rates of $0.20 \%-1.69 \%$ (1:500 to $1: 59$ anesthetics). The five reported errors/pre-errors in PEDS occurred in two

Table 3 Reported medication error rate by surgical case type per anesthetic

\begin{tabular}{lll}
\hline Case Type & Incidence & Percent \\
\hline Neurosurgery & $0 / 372$ & 0.0 \\
Ophthalmology & $0 / 1023$ & 0.0 \\
Off-site Sedation & $2 / 996$ & 0.20 \\
OB/GYN & $5 / 1,589$ & 0.31 \\
ENT & $4 / 976$ & 0.41 \\
General Surgery & $8 / 1,685$ & 0.47 \\
Orthopedics & $9 / 1,602$ & 0.56 \\
Urology & $5 / 801$ & 0.62 \\
CVT & $3 / 299$ & 1.00 \\
Colorectal & $4 / 376$ & 1.06 \\
Transplant & $2 / 179$ & 1.12 \\
Peripheral Vascular & $5 / 380$ & 1.32 \\
Pediatrics & $5 / 296$ & 1.69 \\
Total & $52 / 10,574$ & \\
\hline
\end{tabular}

52 reported errors by surgical specialty from 10,574 anesthesia cases. $\mathrm{OB} / \mathrm{GYN}=$ obstetrics/gynecology; $\mathrm{ENT}=$ otolaryngology; $\mathrm{CVT}=$ cardiovascular/thoracic surgery pediatric urology cases, two pediatric ENT cases, and one pediatric ophthalmology case. Four of the five errors reported were due to incorrect dosing, with the remaining error reported as an omission.

The distribution of medication errors/pre-errors was also analyzed by patient ASA physical status classification. The relative risk of reporting a medication error/pre-error in cases involving ASA III patients $(0.81 \%$ reported incidence) when compared with ASA I $(0.28 \%$ reported incidence) and ASA II patients ( $0.43 \%$ reported incidence) was 2.9:1 and 1.9:1, respectively. This finding was statistically significant (Table 4).

The distribution of cases for provider level of experience was 7,076/10,574 (66.9\%) for the experienced provider group and 3,498/10,574 for the anesthesia provider-intraining group $(33.1 \%)$. The distribution of reported medication errors/pre-errors for type of provider was 26/3,498 ( $0.74 \%$ or $1: 135$ anesthetics) for the anesthesia provider-intraining group and 26/7,076 (0.37\%, or 1:272 anesthetics) for the experienced provider group. Comparison of the two groups resulted in a statistically significant difference $(P=0.0087)$ with a relative risk ratio of 2 (Table 5).

Factors reported as contributing to medication errors/ pre-errors were distraction $(16.7 \%)$, haste/pressure to proceed/stress (production pressure) (12.5\%), misread label of medication vial/ampoule $(12.5 \%)$, unexpected/unfamiliar medication vial/ampoule $(5.6 \%)$, lack of knowledge $(5.6 \%)$, inappropriate medication vial storage $(5.6 \%)$, and inadequate communication $(2.8 \%)$. The other/unspecified reasons $(27.8 \%)$ accounted for the remainder of the positive responses (Table 6).

The types of medication errors/pre-errors are shown in Table 7. The two largest categories of medication errors/ pre-errors, incorrect dose and substitution, accounted for $61.5 \%$ of errors, while omission and repetition/insertion accounted for $19.2 \%$ and $17.3 \%$, respectively (Table 7 ). No intervention was required in 29 patients; 20 patients required reversal or antagonist; one patient required reintubation; one patient required prolonged intubation; and one patient required assisted mask ventilation. No deaths or irreversible harm occurred. Twenty-four errors/pre-errors were considered to cause no patient harm, whereas 15 were considered to cause minor morbidity, and 13 were considered to cause major morbidity or prolonged hospitalization.

One error of unattended epidural infusion medication was also reported $(1.9 \%)$. Sedatives and opioids were reported in $47.4 \%$ of incorrect dose errors; steroids (dexamethasone), and vasoactive medications (phenylephrine) were involved in $46.2 \%$ of substitution errors. The remaining reports $(5.8 \%)$ involved epidural infusions, with one due to an incorrect dose and the remaining due to an unattended infusion bag (Table 8). 
Table 4 Medication Errors Reported and Number of Anesthetics by Patient ASA Classification

\begin{tabular}{lccllll}
\hline ASA & \multicolumn{2}{l}{ Classification $(n=52)$} & & \\
\cline { 2 - 7 } & $\begin{array}{l}\text { Errors } \\
\text { Reported }\end{array}$ & $\begin{array}{l}\text { \% of Total Reported } \\
\text { Errors }(n=52)\end{array}$ & $\begin{array}{l}\text { Number }(\%) \text { of Total } \\
\text { Anesthetics }(n=10,574)\end{array}$ & $\begin{array}{l}\text { Errors Reported/Total ASA } \\
\text { Class Anesthetic }\end{array}$ & $\begin{array}{l}P \text { value when } \\
\text { compared with ASA I }\end{array}$ & $\begin{array}{l}\text { Self-reported } \\
\text { Error Rate }\end{array}$ \\
\hline I & 5 & 9.6 & $1,797(17.0)$ & $5 / 1,797(0.28 \%$ or $1: 359)$ & N/A & $0.05 \%$ \\
II & 24 & 46.2 & $5,583(52.8)$ & $24 / 5,583(0.43 \%$ or $1: 233)$ & 0.2563 & $0.23 \%$ \\
III & 22 & 42.3 & $2,712(25.6)$ & $22 / 2,712(0.81 \%$ or $1: 123) *$ & $0.0158^{*}$ & $0.21 \%$ \\
IV & 1 & 1.9 & $461(4.4)$ & $1 / 461(0.22 \%$ or $1: 461)$ & 0.7464 & $0.01 \%$ \\
V & 0 & 0.0 & $16(0.2)$ & $0 / 16(0.0 \%)$ & 1.0000 & 1.0000 \\
VI & 0 & 0.0 & $5(0.0)$ & $0 / 5(0.0 \%)$ & $0.0 \%$ & $0.0 \%$ \\
\hline ASA
\end{tabular}

$\mathrm{ASA}=$ American Society of Anesthesiologists physical status. N/A = not available

Table 5 Medication errors reported and number of anesthetics by experience of provider

\begin{tabular}{lllll}
\hline $\begin{array}{l}\text { Anesthesia } \\
\text { Provider } \\
\text { Group }\end{array}$ & $\begin{array}{l}\text { Errors } \\
\text { Reported }\end{array}$ & $\begin{array}{l}\% \text { of } \\
\text { Total } \\
\text { Reported } \\
\text { Errors } \\
(n=52)\end{array}$ & $\begin{array}{l}\text { Number }(\%) \\
\text { of Total } \\
\text { Anesthetics } \\
(n=10,574)\end{array}$ & $\begin{array}{l}\text { Self-reported } \\
\text { Error Rate } \\
\text { (Incidence) }\end{array}$ \\
\hline $\begin{array}{l}\text { Providers-in- } \\
\quad 26\end{array}$ & 50.0 & $3,498(33.1)$ & $0.74 \%(1: 135)$ \\
$\quad \begin{array}{l}\text { Training } \\
\text { Group }\end{array}$ & & & & \\
$\begin{array}{l}\text { Resident } \\
\text { SRNA }\end{array}$ & 25 & 48.1 & & \\
$\begin{array}{l}\text { Experienced } \\
\quad \text { Provider }\end{array}$ & 26 & 50.0 & $7,076(66.9)$ & $0.37 \%(1: 272)$ \\
$\quad$ Group & & & & \\
Attending & 3 & 5.8 & & \\
$\quad$ Physician & & & & \\
CRNA & 23 & 44.2 & & \\
Total Errors & 52 & 100.0 & $10,574(100.0)$ & \\
$\quad$ Reported & & & & \\
\hline
\end{tabular}

$\overline{\text { SRNA }=\text { student } \text { nurse anesthetists; } \text { CRNA }=\text { certified registered }}$ nurse anesthetists

\section{Discussion}

This study extended the landmark articles of Webster et $a{ }^{1}{ }^{1}$ and Llewellyn $e t a l{ }^{3}$ to include the role of type of surgical case, severity of medical comorbidities, and level of experience of anesthesia provider on the incidence of medication error/pre-error. The strengths of our study include the collection of data prospectively over a sixmonth time period and inclusion of negative responses to allow for the collection of an accurate denominator from which to calculate response rates. Like Webster et al. and Llewellyn et al., ${ }^{1,3}$ we adopted a conservative approach by using the total number of anesthetics administered during the study period rather than the number of forms returned. This approach allowed the calculation of the minimum incidence of medication errors/pre-errors. Webster et al.
Table 6 Factors leading to errors/pre-errors

\begin{tabular}{lcc}
\hline Factors Leading to Error/Pre-Error & Number & Percent \\
\hline Distraction & 12 & 16.7 \\
Haste/pressure to proceed/stress & 9 & 12.5 \\
Misread label/look-alike vial or ampoule & 9 & 12.5 \\
Unexpected/unfamiliar vial or ampoule & 4 & 5.5 \\
Lack of knowledge/experience & 4 & 5.5 \\
Inappropriate vial storage (vial in wrong location) & 4 & 5.5 \\
Equipment not available & 2 & 2.7 \\
Timing/readjustment of inhalation agents & 2 & 2.7 \\
Infusion pump misprogramming/equipment & 2 & 2.7 \\
$\quad$ malfunction & & \\
Inadequate communication & 2 & 2.7 \\
Inadequate patient information (allergy, etc) & 1 & 1.3 \\
Infusion line disconnection & 1 & 1.3 \\
Sound-alike & 0 & 0.0 \\
Fatigue & 0 & 0.0 \\
Infusion too fast & 0 & 0.0 \\
Other/unspecified & 20 & 27.7 \\
\hline
\end{tabular}

first reported the frequency and nature of drug administration errors during the conduct of anesthesia using these methods, ${ }^{1}$ and in 2009 , Llewellyn et al. reported observations similar to the Webster study using similar methods. ${ }^{3}$ The calculation of response rates and minimum incidence of medication errors/pre-errors in our study allowed comparison with baseline for the response rates as well as with reported minimum incidence of medication errors/preerrors established in the Webster and Llewellyn studies. ${ }^{1,3}$ Had we used the number of returned forms as the denominator, the actual error/pre-error rate would have been 1:169 anesthetics, an incidence still similar in comparison with the findings by Webster et al. and Llewellyn et al.

Based on the total anesthetics delivered during the study period, our overall reported error rate was $0.49 \%$ or $1: 203$ anesthetics. This rate is similar to the medication error rates 
Table 7 Types of error/pre-error reported

\begin{tabular}{ll}
\hline Type of Error/Pre-error & Reported Occurrence \\
\hline Incorrect Dose & $36.5 \%$ \\
Substitution & $25.0 \%$ \\
Omission & $19.2 \%$ \\
Repetition & $15.4 \%$ \\
Insertion & $1.9 \%$ \\
Unattended drug & $1.9 \%$ \\
\hline
\end{tabular}

Table 8 Drugs involved in error by type of error

Error Type (type Drug Involved (number) total)

\begin{tabular}{cl}
\hline $\begin{array}{l}\text { Incorrect Dose } \\
(19)\end{array}$ & $\begin{array}{c}\text { fentanyl (5), ephedrine, cefazolin, labetalol (2), } \\
\text { fentanyl/bupivacaine, heparin, propofol, } \\
\text { phenylephrine, fentanyl/hydromorphone, } \\
\text { morphine, midazolam (3), alemtuzumab } \\
\text { Substitution (13) } \\
\text { dexamethasone (3), phenylephrine (3), mannitol, } \\
\text { thiopental, succinylcholine, ticarcillin/ } \\
\text { clavulanate, hydralazine, morphine, ketorolac } \\
\text { cefazolin (3), vancomycin (3), gentamicin, } \\
\text { methohexital, heparin, unspecified (1) }\end{array}$ \\
Omission (10) & $\begin{array}{l}\text { cefazolin (2), betadine, heparin, zolpidem, } \\
\text { pancuronium, neostigmine, other (1) }\end{array}$ \\
Repetition (1) & cefazolin \\
Unattended & mepivacaine/bupivacaine \\
Drugs (1) &
\end{tabular}

reported by Webster et al., ${ }^{1} 0.75 \%$ (1:133 anesthetics); Llewellyn et al., ${ }^{3}$ 0.37\% (1:274 anesthetics); and Yamamoto et al. ${ }^{4} 0.22 \%$ (1:450 anesthetics).

An important finding of our study is our survey instrument response rate of $83.0 \%$. This high response rate may have been due to the development of a non-threatening environment or a systems-based approach to a clinical problem by assuring that all responses would be voluntary and anonymous. Fear of lawsuit or professional incompetence can be avoided, and reporting trivial errors or near misses as well as those that cause harm has the potential to strengthen care processes and improve quality of care afforded patients. ${ }^{11}$ The conduct of medical care in our work environment needs to be safe for us and for our patients, and as such, medical administrators need to provide a "non-threatening" and "systems-based" approach to the clinical practice environment in order to encourage practitioner reporting and sharing of incident information. ${ }^{12}$ This systems-based environment approach to reporting achieved a better survey instrument response rate than was reported by either Webster et al. (72\%) or Llewellyn et al. (53\%). ${ }^{1,3}$ Moreover, this improved reporting rate may also be explained by increased awareness of the need for error reporting and the shift of organizational cultures over the past decade to emphasize safety rather than blame.

With respect to the type of surgical case, we observed that CVT, CR, VASC, TRANS, and PEDS surgical cases had reported medication error/pre-error rates of $>1.0 \%$, equalling an occurrence rate of one in 59-100 anesthetics. Moreover, the adult surgical cases had higher ASA scores, required more multiple drug infusions, and typically required medications infrequently used. When we examined the pediatric surgical cases, which had a reported occurrence of $1 / 59$ (1.69\%), incorrect dosing was found to be the most common reported event. This observation is consistent with published data on medication errors in pediatric patients. ${ }^{13,14}$ Incorrect dosage errors in pediatric surgery are a well-recognized problem as the frequent need for weight-adjusted dosing makes these patients particularly susceptible to this type of error. Our findings support the relationship between the type of surgical case and the incidence of medication errors/pre-errors.

American Society of Anesthesia physical status classification is used to rank patients objectively based on the severity of their medical comorbidities. ${ }^{15} \mathrm{We}$ found a statistically significant difference in the voluntarily reported frequency of medication errors/pre-errors during surgical cases involving ASA class III patients when compared with ASA class I or II patients. However, no statistically significant difference was found in cases involving ASA IV, $\mathrm{V}$, or VI patients. The lack of statistical significance may be due to the small number of these cases observed in our series. Certainly, a study examining these complex cases is warranted but may require multicentre clinical trials or access to large databases to obtain sufficient data for statistical analysis.

We also observed a significant difference in the reporting patterns and the reported incidence of medication errors/pre-errors when evaluating level of provider experience. Our study showed a twofold increase in the reporting of medication errors/pre-errors by anesthesia providers-in-training compared with experienced providers. This finding is in contrast with the report by Llewellyn et $a l^{3}$ where a higher incidence of error was observed with experienced anesthesia providers.

Factors contributing to medication error/pre-error rates have been previously described. Webster et al. ${ }^{1}$ reported procedural problems, such as failure to check, distraction, inattention, haste, or communication problems, as comprising five of the top seven factors (70\% of errors). Kahn and $\mathrm{Hoda}^{9}$ reported errors divided into categories, i.e., knowledge-based (inadequate knowledge), rule-based (failure to follow set rules to prevent error), and skill-based (inattention, haste, fatigue, stress, or illness). These categories, similar to those described in the Webster ${ }^{1}$ study, accounted for $71.2 \%$ of errors reported. Our findings are 
similar, with six of the top seven factors (61\% of errors) reported as distraction, haste, misread medication label/ vial, lack of knowledge/experience, unexpected/unfamiliar medication vial, and inappropriate medication vial storage. Interestingly, unlike the Webster $^{1}$ or Kahn and Hoda ${ }^{9}$ studies, fatigue was not reported to be a contributing factor in our study. This observation could be due to new residency work hour requirements. Since analysis of these data was from forms with positive responses, we do not know whether fatigue or other human factors played any role in cases with negative responses or those from non-responders. Although reported factors differ slightly, inattention or distraction, haste, and lack of knowledge appear to be consistent when compared with earlier studies. 1,9

The types of medication errors/pre-errors reported in this study are similar to those reported in previous studies. ${ }^{1-4}$ Incorrect dose and substitution errors were the top two reported errors, comprising over half of the errors in our study and comparable with the rates reported by Webster et al., ${ }^{1}$ Bowdle, ${ }^{2}$ Llewellyn et al., ${ }^{3}$ and Yamamoto et $a l .{ }^{4}$ As human error will occur, systems-based methods should be actively studied to minimize medication errors further or hopefully to prevent errors from affecting patients.

A limitation to our study is the requirement of a large sample size to determine statistical significance due to the small incidence of actual medication errors/pre-errors. ${ }^{10}$ This limitation can make the study of medication errors/ pre-errors difficult and expensive, which, in both instances, may inhibit study. Key features of this study were the ongoing reassurance to anesthesia providers in the department that this study was being conducted in a "nonthreatening" environment; responses were voluntary and anonymity was guaranteed; and the data obtained would lead to improved medical care. Another common limitation in the study of medication errors/pre-errors in anesthesia may be related to real-time reporting of events. ${ }^{16,17}$ By design, our reporting survey instrument accompanied every anesthesia record, thus allowing timely and efficient data collection. Continuous re-education of the anesthesia providers was also deemed necessary to encourage voluntary compliance.

The reporting of medication error/pre-error rates in our study is similar to previous studies based on total anesthetics administered. In a recent study, Merry et al. ${ }^{18}$ have reported medication error/pre-error rates based on total administrations per anesthetic. By using an electronic barcode reader system and barcode labelled syringes, they were able to measure automatically the number of administrations of a medication in each anesthetic. This method allowed determination of the rate of medication error/pre-error per administration per anesthetic, which is an important difference. As the authors state, each anesthetic is different, with intravenous boluses, inhalational agents, and intravenous infusions varying among anesthetics. Sicker patients may require more administrations of medications per anesthetic. While we agree with these statements, many hospitals in the United States (including ours) do not currently have an automated system in which to capture this type of information electronically. As our study was completed using facilitated manual reporting forms, the total number of administrations per anesthetic was not captured. While reporting medication errors/pre-errors per administration per anesthetic may be ideal, without the ability to capture each administration automatically, reporting of errors/pre-error rates or establishing a baseline of errors/pre-errors may not yet be achievable in all settings. Until automated solutions and bar-coded labels are available, medication error/pre-error reporting in the United States will be difficult to assess based on per administration per anesthetic, and any attempt at measurement is potentially flawed.

An important factor when carrying out a study with a large sample size where measurement may be potentially flawed is to establish an environment that supports the reporting of errors/pre-errors, and in our specialty, to achieve a change of provider attitudes towards acknowledging and reporting errors/pre-errors. The need for continuous re-education and encouragement to improve voluntary compliance suggests a previous culture that took a more punitive approach to commission or omission of errors than this systems-based approach that leads to enhanced patient safety. In our view, we achieved a change in culture during this study, and future studies may have a similar effect in other institutional settings. Further studies at our institution are warranted to determine whether longterm changes in culture have occurred.

Our reliance on voluntary reporting may have underestimated the true incidence of errors, but as Llewellyn et al. have reported, even a $100 \%$ response rate would underestimate the true incidence because of medication errors/pre-errors that go unrecognized by the anesthesia provider. Observational studies in critical care settings have consistently shown higher incidences with the use of survey instruments rather than with voluntary reporting. ${ }^{19}$ In an editorial, Cohen states, however, that reporting systems should be voluntary as they provide better information for reducing errors. ${ }^{20}$ Most voluntary surveys ${ }^{20,21}$ have reporting rates much less than $100 \%$. We are in agreement with Cohen that voluntary reporting is the best method to collect medication error/pre-error data ${ }^{20}$; however, in our study, only $8,777(83 \%)$ of the total 10,574 anesthetics had sufficient data to be analyzed. Caution should be taken when interpreting these observations as there were 1,797 anesthetics performed without sufficient data. 
The statistically significant difference we observed between the reported medication errors/pre-errors in ASA III patients $v s$ ASA I and ASA II patients are in contrast to Yamamoto et $a l^{4}{ }^{4}$ who retrospectively did not show statistical significance in ASA classification on medication error rates. Whether the difference in study design (prospective $v s$ retrospective) accounted for the difference is unknown. Likewise, Llewellyn et $a{ }^{3}{ }^{3}$ found opposite characteristics of reporting patterns between anesthesia providers-in-training and experienced providers when compared with our observations. However, in a retrospective analysis during the early stages of anesthesia training, Ishikawa et $a .^{22}$ observed that the less experienced the provider, the higher the medication error rates. Due to the small sample size of all of these studies, caution must be taken when applying the results. A larger multicentre study or access to large databases would clarify these observations.

Anonymity was considered of paramount importance in preventing errors in the perioperative period along with the development of a supportive educational environment. Studies that cannot ensure anonymity will underestimate the true incidence of medication errors. As in the Llewellyn study, ${ }^{3}$ anonymity limited our ability to intervene to improve response rate; however, we hypothesize that the ongoing training and encouragement resulted in a higher voluntary response rate in our study when compared with the response rate observed in the Llewellyn study. ${ }^{3}$ Moreover, in a study of anesthesiologists in New Zealand wherein only $12.5 \%$ of anesthesiologists responded to a drug administration error survey, the lack of true anonymity played an important limiting factor in the response rate. $^{23}$ Most medication error studies to date, including ours, suffer from these limitations, and until health care environments can be made fault-tolerant, the true incidence of medication error in our specialty will remain underestimated.

Anesthesiologists are unique among physicians because they prescribe, dispense, compound/prepare, and administer medications, ${ }^{24}$ yet as physicians, they are not trained in traditional medical school programs on how to perform many of these tasks. Nurses are trained in administration and preparation of medications but not in ordering, dispensing, or compounding medication. Pharmacists are trained in compounding, dispensing, monitoring, and intervention of incorrect order of medications. Anesthesia residents and student nurse anesthetists learn these practices "on the job" with little formal education, thus leading to a possible increase in the risk of making a medication error/pre-error. Our observation that anesthesia providersin-training tend to report these errors more often than experienced providers also suggests that more focused education in management and administration is desirable to minimize medication errors/pre-errors during the early part of specialty training. ${ }^{25}$

In conclusion, reports of medication errors/pre-errors continue to occur in the practice of anesthesiology. Although our reporting incidence rates were similar to those of other studies, we also observed that surgical case type, patient ASA classification, and the level of provider experience play a role in the frequency of medication errors/pre-errors in a large academic teaching hospital. As human error cannot be eliminated and as anesthesiologists work in dynamic health care environments, reporting systems that share incident information in a systems-based culture can lead to an environment with enhanced patient safety. $^{12}$

Acknowledgements The authors sincerely thank Alan Merry, Michael Wall, and Joyce Wahr for reviewing this manuscript and also for their helpful advice.

Competing interests None declared.

\section{References}

1. Webster CS, Merry AF, Larsson L, McGrath KA, Weller J. The frequency and nature of drug administration error during anaesthesia. Anaesth Intensive Care 2001; 29: 494-500.

2. Bowdle TA. Drug administration error from the ASA Closed Claims Project. ASA Newsletter 2003; 67: 11-3.

3. Llewellyn RL, Gordon PC, Wheatcroft D, et al. Drug administration errors: a prospective survey from three South African teaching hospitals. Anaesth Intensive Care 2009; 37: 93-8.

4. Yamamoto M, Ishikawa S, Makita K. Medication errors in anesthesia: an 8-year retrospective analysis at an urban university hospital. J Anesth 2008; 22: 248-52.

5. Fasting S, Gisvold SE. Adverse drug errors in anesthesia, and the impact of coloured syringe labels. Can J Anesth 2000; 47: 1060-7.

6. Michaels AD, Spinler SA, Leeper B, et al. Medication errors in acute cardiovascular and stroke patients: a scientific statement from the American Heart Association. Circulation 2010; 121: 1664-82.

7. Abeysekera A, Bergman IJ, Kluger MT, Short TG. Drug error in anaesthetic practice: a review of 896 reports from the Australian Incident Monitoring Study database. Anaesthesia 2005; 60: 2207.

8. Sakaguchi Y, Tokuda K, Yamaguchi K, Irita K. Incidence of anesthesia-related medication errors over a 15 -year period in a university hospital. Fukuoka Igaku Zasshi 2008; 99: 58-66.

9. Kahn FA, Hoda MQ. A prospective survey of intra-operative critical incidents in a teaching hospital in a developing country. Anaesthesia 2001; 56: 177-82.

10. Nyssen AS, Blavier A. Error detection: a study in anaesthesia. Ergonomics 2006; 49: 517-25.

11. Wolf $Z R$, Hughes $R G$. Error reporting and disclosure. In: Hughes RG, editor. Patient Safety and Quality: An Evidence-Based Handbook for Nurses. Rockville, MD: Agency for Healthcare Research and Quality (US); 2008 .

12. Koczmara $C$, Dueck $C$, Jelincic $V$. To err is human, to share is divine. Dynamics 2006; 17: 22-5. 
13. Engum SA, Breckler FD. An evaluation of medication errors-the pediatric surgical service experience. J Pediatr Surg 2008; 43: 348-52.

14. Ghaleb MA, Barber N, Franklin BD, Yeung VW, Khaki ZF, Wong $I C$. Systematic review of medication errors in pediatric patients. Ann Pharmacother 2006; 40: 1766-76.

15. Meyer $S$. Grading of patients for surgical procedures. Anesthesiology $1941 ; 2: 281-4$.

16. Choy YC. Critical incident monitoring in anaesthesia. Med $\mathrm{J}$ Malaysia 2006; 61: 577-85.

17. Rozich JD, Haraden CR, Resar RK. Adverse drug event trigger tool: a practical methodology for measuring medication related harm. Qual Saf Health Care 2003; 12: 194-200.

18. Merry AF, Webster CS, Hannam J, et al. Multimodal system designed to reduce errors in recording and administration of drugs in anaesthesia: prospective randomised clinical evaluation. BMJ 2011; 343: d5543.

19. Cullen DJ, Bates DW, Adverse Drug Event Prevention Study Group. Prevention of adverse drug events: a decade of progress in patient safety. J Clin Anesth 2000; 12: 600-14.
20. Cohen MR. Why error reporting systems should be voluntary. BMJ 2000; 320: 728-9.

21. Orser BA, Byrick $R$. Anesthesia-related medication error: time to take action. Can J Anesth 2004; 51: 756-60.

22. Ishikawa S, Murai K, Yokoyama K, Masuda A, Nakazawa K, Makita $K$. Retrospective analysis on misapplication of anesthesiarelated drugs in the early stage of anesthesia training. J Anesth 2006; 20(Suppl): 265.

23. Merry AF, Peck DJ. Anaesthetists, errors in drug administration and the law. N Z Med J 1995; 24: 185-7.

24. Wheeler SJ, Wheeler DW. Medication errors in anaesthesia and critical care. Anaesthesia 2005; 60: 257-73.

25. Jensen LS, Merry AF, Webster CS, Weller J, Larsson L. Evidence-based strategies for preventing drug administration errors during anaesthesia. Anaesthesia 2004; 59: 493-504. 